\title{
Formación en técnicas para la innovación en trabajo social y educación social. Una experiencia interuniversitaria colaborativa
}

\author{
Isabel Torras ${ }^{1}{ }^{\text {}}$, Eva M. Rubio², Rosalía Mota ${ }^{2}$ y Santa Lázaro \\ (1) Facultad de Educación Social y Trabajo Social Pere Tarrés, Universidad Ramón Llull. Barcelona-España \\ (2) Facultad de Ciencias Humanas y Sociales. Universidad Pontificia Comillas. Madrid-España. \\ (Correo - e: itorras@peretarres.org; erubio@comillas.edu; rmota@comillas.edu; slazaro@comillas.edu)
}

*Autor a quién debe ser dirigida la correspondencia

Recibido Abr. 8, 2021; Aceptado Jun. 7, 2021; Versión final Jun. 26, 2021, Publicado Oct. 2021

\begin{abstract}
Resumen
Se presenta una experiencia formativa de innovación docente con estudiantes y profesores de los grados de Trabajo Social y Educación Social de las Universidades Ramón Llull, Deusto y Comillas (España), en el curso 2019/20. El objetivo fue generar propuestas innovadoras y útiles para la praxis profesional en el acompañamiento a personas en sus transiciones vitales. Se utilizaron dinámicas de diseño creativo, trabajándose colaborativamente sus cinco fases: empatizar, definir, idear, crear y evaluar. La satisfacción con la experiencia se midió con un cuestionario administrado online, al que respondieron el $82,3 \%$ de los estudiantes. Destacan como contribuciones especialmente valiosas la autopercepción de competencias de inspiración, narración y representación de proyectos innovadores; el desarrollo de la creatividad y el pensamiento crítico; el trabajo colaborativo y la relación horizontal entre estudiantes y profesores en un contexto interuniversitario; y el fortalecimiento de la motivación por seguir aprendiendo. Se concluye que las técnicas formativas propuestas son percibidas muy positivamente por los estudiantes, abriéndoles nuevas miradas para su ejercicio profesional.
\end{abstract}

\section{Training in techniques for innovation in social work and social education. A collaborative interuniversity experience}

\begin{abstract}
This paper presents a training experience of teaching innovation with students and lecturers from the bachelor's degrees in Social Work and Social Education at the Ramon Llull, Deusto and Comillas Universities (Spain), during the 2019-2020 academic year. The aim was to generate innovative and useful proposals for professional praxis in accompanying people in their life transitions. Creative design dynamics were used, working collaboratively in its five phases: empathizing, defining, devising, creating, and evaluating. The satisfaction with the experience was measured with a questionnaire administered online to which $82,3 \%$ of the students responded. Particularly valuable contributions include the self-perceived competences in inspiration, narration and representation of social projects; the development of creativity and critical thinking; the collaborative work and horizontal relationship between students and teachers in an inter-university context; and the strengthening of the motivation to continue learning. It is concluded that the proposed training techniques are perceived very positively by students, opening up new perspectives for their professional practice.
\end{abstract}




\section{INTRODUCCIÓN}

La sociedad actual se define por el cambio vertiginoso y global. Ha desaparecido el modelo tradicional de las relaciones socioeconómicas, las relaciones personales y los itinerarios vitales. Vivimos pues, en una sociedad "líquida" (Bauman, 2009) y en constante evolución, lo que impide predecir e identificar tendencias y ajustar el comportamiento al porvenir inmediato, empuja a la precariedad y requiere de constantes mutaciones y sucesión de nuevos comienzos. Los contextos en los que los individuos desarrollan sus vidas han dejado de estar estandarizados, definidos y ordenados, para transformarse en lo que se ha dado en denominar entornos VICA, acrónimo que representa las iniciales de sus cuatro rasgos predominantes: Volatilidad, referida a la alta velocidad del cambio y por consiguiente a la necesidad de tomar decisiones con rapidez; Incertidumbre ante el porvenir, sin capacidad para predecir el futuro inmediato; Complejidad derivada del alto número de factores y de relaciones entre ellos que es preciso tener en cuenta, y Ambigüedad en la interpretación de lo que nos rodea cuando la información es incompleta, contradictoria o poco fiable. Estos rasgos se relacionan entre sí, influyéndose mutuamente e implicando una creciente dificultad en el análisis y la toma de decisiones en estos escenarios (Bennet y Lemoine, 2014; Millar et al., 2018).

La reciente incidencia de la pandemia de Covid-19, y su impacto en la percepción de incertidumbre y vulnerabilidad en múltiples facetas de la vida de las personas simultáneamente en todas partes del mundo, no ha hecho sino intensificar y acelerar este proceso poniendo en evidencia las grietas de una sociedad que afronta nuevos e impactantes desafíos que no admiten espera (Sathiabalan et al., 2020). Este escenario pone en cuestión las bases y formas tradicionales de la acción social y su capacidad para ajustarse y actuar frente a las nuevas dificultades de los individuos, que quedan cada vez más expuestos a la deriva de las circunstancias, sin momento de reposo ni seguridad. De esta manera, la intervención social debe vincularse al acompañamiento de las personas en sus trayectorias de vida que son inciertas, complejas y fragmentadas, especialmente en los colectivos más desfavorecidos, para los que las transiciones vitales son aún más inestables, indeterminadas y problemáticas. Las profesiones del Trabajo Social y la Educación Social han de asumir este reto creando condiciones que posibiliten a las personas transitar por los procesos de cambio que se producen en sus vidas con la seguridad y los apoyos suficientes. Para ello, es prioritario que los futuros profesionales, durante su formación universitaria, incorporen una perspectiva innovadora y creativa en el análisis y la respuesta ante las dificultades que pueden darse al afrontar distintas transiciones vitales. (Staniforth et al., 2011; Leal et al., 2016).

La innovación social representa una oportunidad para que las organizaciones de intervención social puedan responder de forma creativa y útil a problemas sociales crecientes, adaptando sus servicios a las necesidades reales de las personas. Delimitar el concepto de innovación social es una tarea compleja (Hernández-Ascanio et al., 2016). Se define como el conjunto de actividades y servicios dirigidos a responder a una necesidad social y que, en su desarrollo, crean nuevas relaciones, fortalecen la capacidad social de actuación y, generalmente, son compartidas y difundidas por individuos y organizaciones sociales (Mulgan 2006, 2019; Martínez Moreno et al, 2019).

La innovación social incorpora tres aspectos principales intensamente relacionados entre sí y que se influyen mutuamente de forma positiva (Howaldt, 2019): a) tratar de responder a los principales retos y problemas sociales mediante políticas globales y sistémicas que incorporen el conocimiento emergente, dirigidas a resolver problemas que las personas han de afrontar en su vida cotidiana, b) dar un mayor reconocimiento a las innovaciones no tecnológicas orientadas a cambiar las prácticas sociales y c) diseñar prácticas y procesos de innovación abiertos a la sociedad. Desde este punto de vista, el Trabajo Social y la Educación Social se han convertido en sectores cruciales para la innovación, tanto por sus intereses y objetivos como por su enfoque conceptual y metodológico. Además, ambas profesiones son, en sí mismas, un ejemplo de innovación tanto desde sus orígenes, en su empeño por responder a dificultades y necesidades sociales, como por su constante evolución para responder a los problemas sociales siempre cambiantes (Nandan et al., 2015; Alonso y Echevarría, 2016). Como señalan Edwards-Schachter et al. (2017), esta necesidad de innovación puede considerarse como una obligación para estas profesiones.

La universidad es el escenario clave para que los estudiantes, como futuros profesionales, adquieran las competencias necesarias para trabajar con perspectiva de innovación social. Por ello, existen numerosas experiencias de universidades que incorporan este enfoque en sus programas formativos, aunque con un desarrollo desigual (Alden et al., 2015). Suelen incidir en la adquisición de conocimientos sobre innovación, el desarrollo de pensamiento crítico y el análisis de procesos de intervención desde distintas perspectivas (Garthwait, 2015), pero no es muy habitual que aborden competencias específicas de creatividad, innovación, diseño y utilización de tecnologías, entre otras posibles. Como consecuencia, es necesario revisar las metodologías docentes utilizando nuevos recursos y técnicas que completen estas carencias como puede ser las técnicas de gamificación, coaching, mentoría, experiencias de aprendizaje-servicio y, en general, metodologías participativas en las que el alumnado tome un papel activo y crítico y que combinen los 
contenidos y las metodologías docentes con el objetivo de motivarle hacia la innovación social (Alonso y Fernández, 2011; Leal et al., 2019).

Un ejemplo de ello es el "Design Thinking", un método de aprendizaje que utiliza el trabajo en equipo para resolver problemas complejos a través de un análisis en profundidad sobre la percepción del problema y las diversas vías de solución (Brown, 2008). Contribuye al desarrollo de competencias clave para la innovación social: pensamiento crítico y solución de problemas, trabajo colaborativo y liderazgo, agilidad y adaptabilidad, iniciativa y actitud emprendedora (Soria-Barreto et al., 2016), comunicación eficaz, acceso y análisis de la información y curiosidad e imaginación.

Esta metodología es eficaz en todos los grupos de edad y en todas las disciplinas y profesiones ya que los participantes han de pensar cómo lo haría un "diseñador", utilizando sus capacidades para concebir, planificar y elaborar un producto, en cualquier área de conocimiento. Esto supone afrontar problemas mediante la generación de ideas, su evaluación y su mejora. Realizar este proceso prepara a los estudiantes para afrontar los complejos problemas de la vida real y proponer soluciones eficaces y responsables (Melles et al, 2012). Desde la perspectiva del profesorado, ofrece un proceso formalizado en diversas etapas que apoya y da estructura al proceso de aprendizaje de las competencias de innovación social. Las fases del proceso son las siguientes: entender o empatizar; observar; sintetizar; idear; crear un prototipo, y, por último, ponerlo a prueba. Además, al tratarse de una experiencia de trabajo en equipo, los profesores se relacionan con los estudiantes de forma más libre y personalizada, lo que contribuye a transmitirles confianza en sus capacidades y a promover la expresión de ideas y el conocimiento compartido. Esta técnica se ha utilizado como metodología docente en la experiencia que se describe en el presente trabajo.

En el marco del consorcio universitario Aristos Campus Mundus (ACM) entre la Universidad de Deusto, la Universidad Ramón Llull y la Universidad Pontificia Comillas, un grupo de docentes realizaron el proyecto de investigación "Los procesos de transición vital como escenarios para la innovación en Trabajo Social y Educación Social" (ACM2018_20, 2018-2019). Se identificaron algunas transiciones y micro-transiciones vitales emergentes a las que responder desde la acción social. Con el objetivo de generar sinergias entre la investigación y la docencia universitaria, este mismo grupo ha desarrollado, a partir de los resultados obtenidos, un proyecto de innovación docente: "Aprender desde las fronteras. Proyecto de desarrollo de competencias para la innovación en Trabajo Social y Educación Social desde las transiciones vitales" (ACM2018innov_05, 2018-2020). Partiendo de los principales ámbitos de transición identificados en la investigación: a) crianza y familia, b) empobrecimiento y empleo y c) pérdidas y duelo, se llevó a cabo un proceso docente en el que los estudiantes aprendieran sobre las transiciones vitales y sus repercusiones en la vida de las personas, reflexionando y proponiendo diversas formas de intervención profesional en estos procesos. Para ello, se utilizaron técnicas de "Design Thinking", que promueven la creatividad y proporcionan una metodología experimental y constructiva para abordar necesidades en los contextos sociales, con un enfoque centrado en las personas (Berzin y Catsouphes, 2015), así como muros colaborativos, y elaboración de prototipos que permitieran el flujo de los procesos de innovación social: empatizar, definir, idear, crear y evaluar.

La finalidad era ofrecer una formación piloto que permitiera a los estudiantes vivenciar una nueva forma de mirar la realidad desde el pensamiento creativo, generando ideas útiles para la praxis profesional en el acompañamiento a las personas que transitan por algunos procesos de cambio en su vida con relación a tres grandes transiciones vitales: la crianza y la familia, el empobrecimiento y el empleo, y las pérdidas y los procesos de duelo. En base a este planteamiento, los resultados de aprendizaje esperados fueron: 1) desarrollo de recursos y estrategias para el aprendizaje y la intervención profesional; 2) ampliación de conocimientos sobre las transiciones vitales; 3 ) autopercepción de adquisición de competencias vinculadas a la innovación, el pensamiento crítico y la creatividad; 4) capacidad de reflexión sobre la propia profesión y las necesidades sociales emergentes, y 5) experimentación de trabajo colaborativo intra e interuniversitario.

\section{MÉTODO}

En esta experiencia estuvieron implicados 16 profesores y 78 alumnos de los Grados de Trabajo Social y Educación Social de las tres universidades. De la Universidad Ramón Llull, Facultad de Educación Social y Trabajo Social Pere Tarrés, participaron 5 profesores y 9 alumnos; de la Universidad Pontificia Comillas, Facultad de Ciencias Humanas y Sociales, se involucraron 4 profesoras y 17 alumnos; y de la Universidad de Deusto, Facultad de Psicología y Educación, participaron 7 profesores y 52 alumnos.

El proyecto partía de la posibilidad del trabajo en red y del intercambio de perspectivas entre el alumnado de las tres universidades, con profesionales y con profesorado de contextos diferentes, pero la realidad de la 
pandemia por Covid-19 obligó a limitar dicho intercambio por los ajustes que debieron hacerse durante el confinamiento domiciliario que se dio en España entre los meses de marzo y mayo de 2020.

Previamente al desarrollo de la experiencia, el profesorado implicado en la misma recibió un seminario de formación presencial sobre las técnicas de diseño creativo que se iban a utilizar, experimentando cada una de las fases que posteriormente se llevarían a cabo en los centros con los estudiantes. Esta formación práctica cumplió con un doble objetivo: familiarizar a todos los implicados con las estrategias de capacitación, y consensuar vivencialmente las nuevas técnicas a transmitir posteriormente en los grupos creados en las distintas Facultades, asegurando los mismos parámetros para su desarrollo. La implementación del proyecto con el alumnado se realizó a lo largo de 5 fases, más una fase 0 o fase previa, en las que se aplicaron técnicas específicas para el desarrollo del pensamiento crítico, la creatividad y el trabajo colaborativo, tal y como se explica a continuación.

Fase 0. Configuración de los grupos de participantes y arranque de la formación. En la Universidad Ramón Llull y en la Universidad Pontificia Comillas la experiencia formativa se planteó como una actividad extracurricular de participación voluntaria por parte de los estudiantes, mientras que en la Universidad de Deusto se incorporó la experiencia en el desarrollo de una de las asignaturas del currículum. El día de inicio de la experiencia formativa, profesorado y alumnado de las diferentes universidades se pusieron en contacto a través de una videoconferencia en la que se clarificaron aspectos prácticos del proceso que se iba a seguir. Esta fase previa a las sesiones propiamente formativas, sirvió para incrementar la conciencia del proyecto común entre el alumnado de las diferentes universidades, aumentando su motivación y vinculación con el mismo. Desde el inicio del proceso formativo se abrieron tres espacios virtuales a través de un muro colaborativo para compartir los "productos" (imágenes, videos o textos) de cada grupo temático (crianza y familia, empobrecimiento y empleo, y pérdidas y duelo), como puede verse en la figura 1.

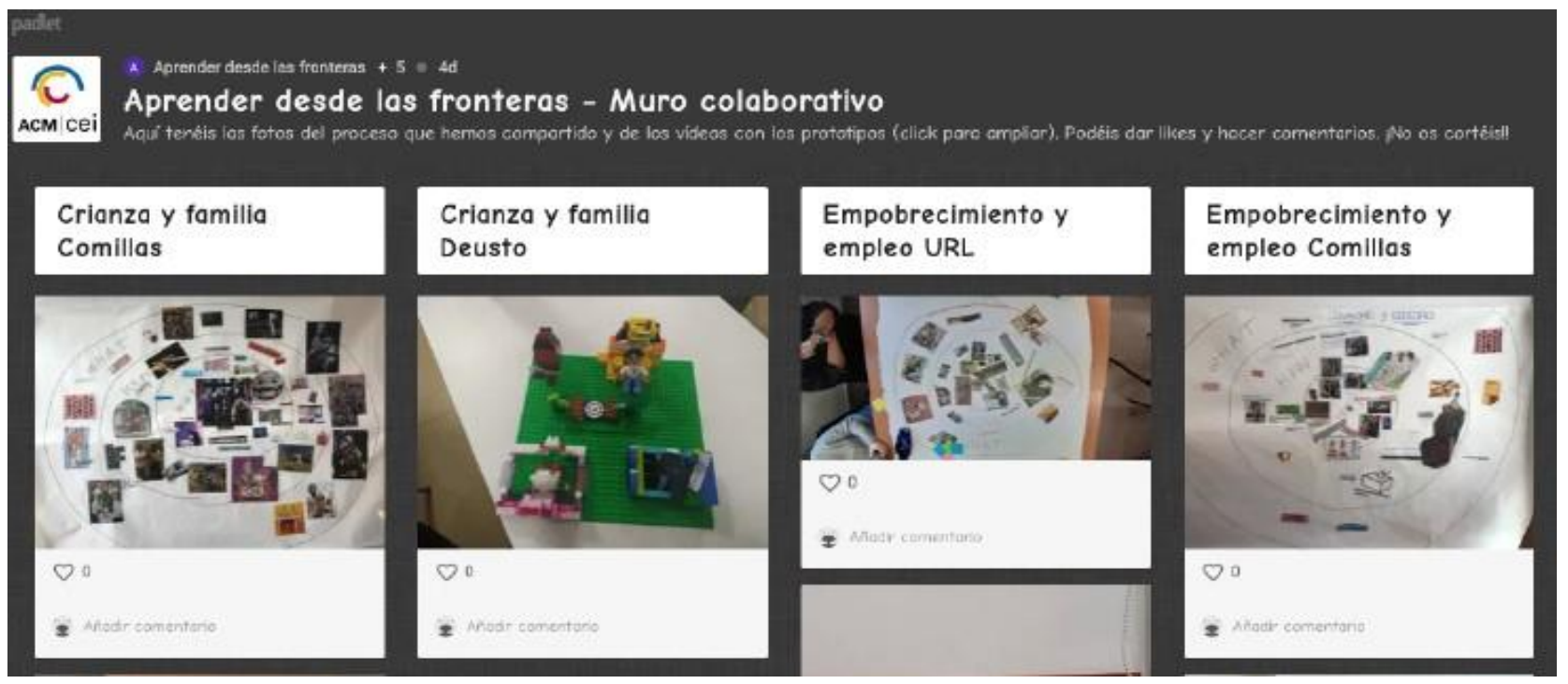

Fig. 1. Muro colaborativo

Fase 1. Empatizar. En esta primera fase, desde el marco de las soluciones intentadas en la construcción de los problemas humanos complejos (Holmberg, 2000), se realizó una dinámica para empatizar con las personas que viven transiciones relativas a la crianza y la familia, el empobrecimiento y el empleo, y las pérdidas y el duelo. Se pidió a los estudiantes que se pusieran en la piel de dichas personas y, mediante la técnica de "cómo empeorar" (Nardone, 2019), pensaran en actuaciones (o en omisiones) que implicaran un empeoramiento de estas situaciones.

Fase 2. Definir. Del listado de actuaciones obtenido en la fase 1 se identificaron cuáles de ellas se llevaban a cabo en las intervenciones profesionales actuales. La técnica consistió, por tanto, en revisar las actuaciones una a una, destacar aquellas que se estaban realizando a pesar de que empeoraban la realidad atendida, y argumentar por qué se consideraba así. Esta actividad permitió comprender cómo las buenas intenciones no son garantía de éxito en la intervención socioeducativa, y sentó las bases para reducir las resistencias al cambio de algunas prácticas profesionales habituales. También ayudó a los estudiantes a evidenciar y romper inercias de las intervenciones sociales. A partir de aquí se identificaron entre 1 y 3 problemas para cada área 
de transición vital, abordados desde la perspectiva de las soluciones con la técnica de la "varita mágica", consensuando la prioridad de intervención.

Fase 3. Idear. En esta fase se utilizó la técnica del "Golden Circle" (Sinek, 2009) para que los estudiantes generaran propuestas sobre las posibles innovaciones a desarrollar. Esta técnica implica que, antes de proponer una idea innovadora en el ámbito social, es necesario aclarar el porqué de su implementación, posteriormente se debe explicar cómo dicha idea innovadora produciría un cambio, haciendo referencia a los métodos, formas y medios y, finalmente, se describe el producto innovador que se desea llevar a cabo. Esta estructura se representa visualmente en la figura 2.

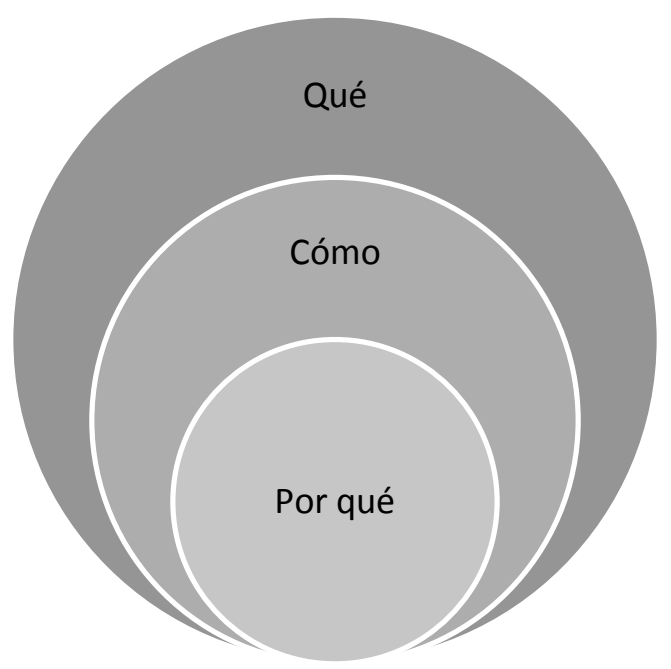

Fig. 2. Diagrama "Golden Circle" (Adaptado de Sinek, 2009)

Cada estudiante, previa reflexión, representó sus ideas innovadoras en un mural por medio de la técnica del Collage, elaborando un relato con el que poder compartir con los compañeros de su grupo el sentido de las imágenes elegidas. La puesta en común de los relatos permitió consensuar, dentro de cada equipo, una estructura narrativa sobre las motivaciones intrínsecas para la innovación, las metodologías a emplear y los productos o servicios a desarrollar (Rosa et al., 2014). Dichas estructuras narrativas se compartieron posteriormente con los otros grupos.

Fase 4. Crear. En los grupos de trabajo de los estudiantes en cada universidad, se crearon prototipos que representaban de manera real o metafórica una propuesta innovadora para cada uno de los tres ámbitos de transiciones vitales abordados: a) crianza y familia, b) empobrecimiento y empleo, y c) pérdidas y duelo. Mediante la utilización de piezas de construcción, plastilina y figuras articuladas, construyeron maquetas que representaban su proyecto (Figura 3). Como rasgos generales, los proyectos se caracterizaron por enfatizar el papel del empoderamiento, la auto-gestión y el establecimiento de redes de apoyo mutuo en el afrontamiento de la transición vital, con los profesionales de la acción social desarrollando roles de acompañamiento y soporte técnico.

A modo de ejemplo, cabe señalar proyectos tales como la creación de una "Red de Crianzas Compartidas" en el ámbito de la crianza y familia, en el que los estudiantes proponen crear un Observatorio IOI (Investigación, Observación e Innovación) de intervención integral y dinámica, que supere la visión tradicional en la atención familiar y en el que las familias comparten sus experiencias y se co-responsabilizan del cuidado de los menores. En el ámbito del empobrecimiento y empleo, el proyecto de creación del "Espacio de Salud, Educación y Trabajo y el Museo de las Puertas Cerradas" propone trabajar el empoderamiento y la capacitación y dejar atrás, cerrando las puertas, las barreras que impiden la incorporación laboral. En el ámbito de las pérdidas y el duelo, un ejemplo es la creación de un "Espacio Abierto: de Gusano a Mariposa" en el que se acompaña a las personas que transitan por las distintas etapas del proceso de duelo y se fomenta la expresión de sus sentimientos de tristeza con el apoyo de otras personas que han vivido experiencias similares de duelo y del acompañamiento de los profesionales.

Tras la construcción de las maquetas de los prototipos, se pidió a los estudiantes que ejercitaran la narrativa exponiendo al resto de los grupos su proyecto y cómo se llevaría a cabo: la relación e interacción entre profesionales, las personas atendidas, la organización y la comunidad; el desarrollo de la intervención, y el acceso a los servicios entre los aspectos más importantes. 


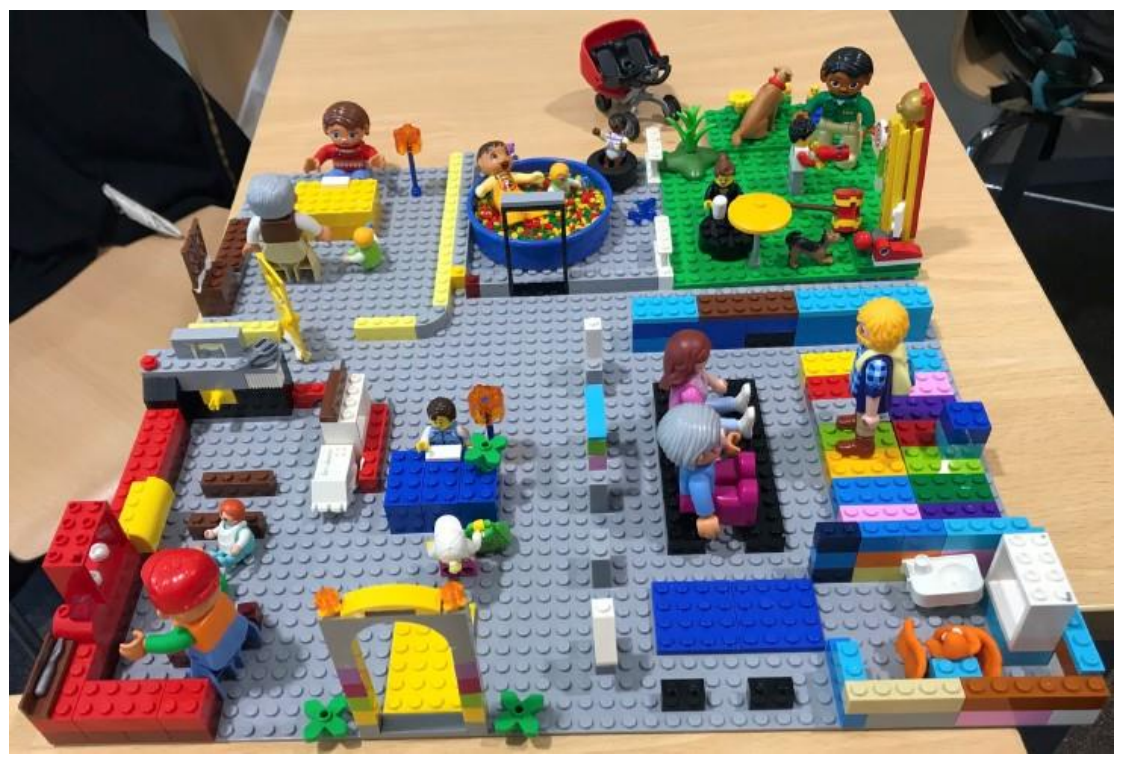

Fig. 3. Prototipo de proyecto innovador

Fase 5. Evaluar: Al finalizar las diferentes fases del proyecto y la aplicación de las diferentes técnicas para la promoción de procesos de innovación social, se crearon espacios de evaluación para reflexionar con el alumnado en torno a lo aprendido, la utilidad de las propuestas y los elementos que podrían ser mejorados. Para ello se utilizaron técnicas diversas: desde el debate constructivo, a la metáfora del objeto en que cada participante debía recurrir a un elemento para representar simbólicamente el aprendizaje realizado. En todos los casos, cada equipo validó su prototipo sustentándolo frente a los compañeros de los otros equipos, argumentando las dimensiones y componentes fundamentales de su propuesta. El alcance del proyecto, por lo tanto, fue el diseño del prototipo y el análisis de sus características principales que se representaron para dar solución a la problemática planteada. Aunque los prototipos no pasaron una validación profesional, los estudiantes fueron capaces de pensar y crear nuevos emprendimientos valiosos para la práctica de su profesión. Es decir, más allá de lograr o no la viabilidad de un prototipo, la experiencia formativa propuesta planteaba, como resultado principal, la mejora del aprendizaje de los estudiantes para responder creativamente a dificultades asociadas a las transiciones vitales en entornos socialmente complejos. La figura 4 refleja las fases realizadas para el desarrollo de la experiencia "Aprender desde las Fronteras":
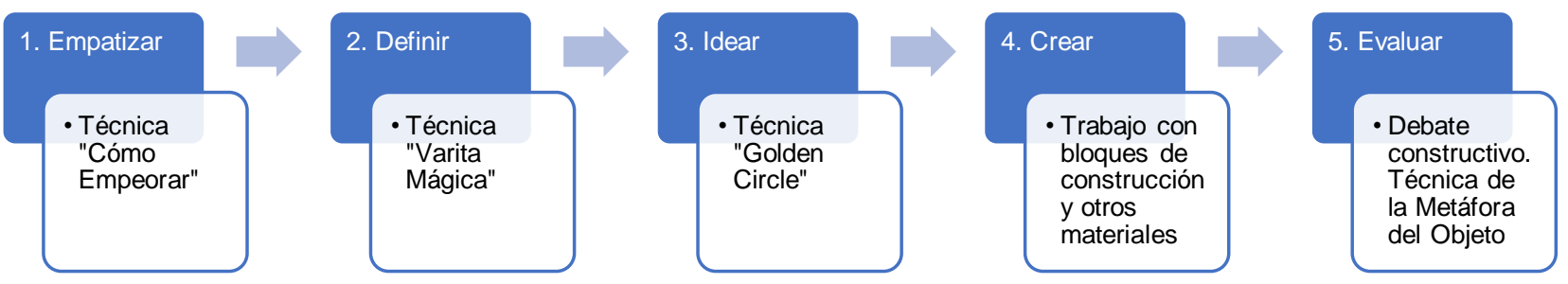

Fig. 4. Fases de "Aprender desde las Fronteras"

Por último, se realizó una evaluación individual y anónima mediante un diseño transversal, utilizándose como instrumento de medición un cuestionario on-line, con el objetivo de recabar la percepción de los estudiantes en relación con los resultados de aprendizaje esperados. El cuestionario, elaborado ad-hoc, contenía 16 preguntas cerradas de escala, en las que los estudiantes evaluaban del 1 al 5 diferentes aspectos de la experiencia, representando el 1 la evaluación más baja y el 5 la más alta. Se añadieron tres preguntas abiertas de carácter cualitativo que indagaban sobre los aspectos más valiosos de la experiencia, las mejoras que introducirían y las tres palabras que asociarían a la innovación. Respondieron al cuestionario 65 estudiantes, lo que significa una tasa de respuesta del $83,3 \%$.

Las áreas evaluadas fueron cinco: el valor de aprendizaje de la experiencia, el conocimiento de las transiciones vitales, el grado en el percibían que habían adquirido competencias de innovación social, la motivación por el aprendizaje y la disposición profesional, y las relaciones con compañeros y profesores durante la experiencia. Dentro del bloque del valor de aprendizaje de la experiencia, se preguntó por la utilidad de la experiencia para el aprendizaje global de los estudiantes y en qué medida ésta les había proporcionado nuevos recursos y estrategias para la intervención profesional. Respecto al conocimiento de las transiciones vitales, los estudiantes valoraron si tenían más conocimientos sobre crianza y familia, empobrecimiento y 
empleo, y pérdidas y duelo, y si la experiencia les había hecho más conscientes de la importancia de las transiciones vitales en la vida de las personas. Entre la percepción de logro de competencias de innovación social por las que el cuestionario preguntaba estaban el cambio de mirada sobre la realidad social, la mejora en general de éstas, y el desarrollo del pensamiento crítico, la creatividad, el trabajo colaborativo y la capacidad de comunicación.

Los estudiantes también evaluaron, dentro del bloque de la motivación por el aprendizaje y la disposición profesional, en qué grado haber formado parte del proyecto les había motivado a seguir aprendiendo y a saber más sobre innovación, y por otra parte les había permitido reflexionar sobre su profesión. Por último, dentro del bloque de las relaciones con otros, se indagó sobre si la experiencia había mejorado la relación con sus compañeros y los profesores implicados en cada universidad, y también su valoración sobre su carácter interuniversitario. En la tabla 1 se resumen las áreas de evaluación de la experiencia y las dimensiones que agrupa cada una de ellas.

Tabla 1: Áreas y dimensiones evaluadas

\begin{tabular}{|c|c|}
\hline Área & Dimensión \\
\hline \multirow{2}{*}{$\begin{array}{l}\text { A1. Valor de aprendizaje de la } \\
\text { experiencia }\end{array}$} & Utilidad de la metodología para el aprendizaje \\
\hline & II. Aprendizaje de nuevos recursos y estrategias para la intervención profesional \\
\hline \multirow[t]{2}{*}{$\begin{array}{l}\text { A2. Conocimiento de las } \\
\text { transiciones vitales }\end{array}$} & $\begin{array}{l}\text { I. Adquisición de más conocimientos sobre crianza y familia, empobrecimiento } \\
\text { y empleo, y pérdidas y duelo }\end{array}$ \\
\hline & $\begin{array}{l}\text { II. Mayor conciencia sobre la importancia de las transiciones vitales en la vida } \\
\text { de las personas }\end{array}$ \\
\hline \multirow{6}{*}{$\begin{array}{l}\text { A3. Adquisición de } \\
\text { competencias de innovación } \\
\text { social }\end{array}$} & Cambio de mirada sobre la realidad social \\
\hline & II. Mejora global de competencias de innovación social \\
\hline & III. Mejora del pensamiento crítico \\
\hline & IV. Mejora de la creatividad \\
\hline & V. Mejora del trabajo colaborativo \\
\hline & VI. Mejora de la capacidad de comunicación \\
\hline \multirow{3}{*}{$\begin{array}{l}\text { A4. Motivación por el } \\
\text { aprendizaje y disposición } \\
\text { profesional }\end{array}$} & Motivación para saber más sobre innovación social \\
\hline & II. Motivación por seguir aprendiendo \\
\hline & III. Reflexión sobre su profesión \\
\hline \multirow[t]{3}{*}{ A5. Relaciones con otros } & Mejora de la relación con sus compañeros \\
\hline & II. Mejora de la relación con el profesorado implicado \\
\hline & $\begin{array}{l}\text { III. Valoración positiva del trabajo con estudiantes y profesores de otras } \\
\text { universidades }\end{array}$ \\
\hline
\end{tabular}

\section{RESULTADOS}

Globalmente la evaluación de la experiencia ha resultado satisfactoria y ello queda reflejado tanto en las medidas cuantitativas como en las cualitativas. A nivel cuantitativo, la valoración media está entre 3,98 puntos ("Este proyecto me ha ayudado a cambiar la mirada sobre la realidad social") y 4,34 puntos ("Este proyecto ha ampliado mi motivación por seguir aprendiendo"), lo que traduce un grado de satisfacción bastante elevado y también una destacable homogeneidad en las valoraciones. En general, son reseñables las bajas desviaciones típicas que tienen todos los aspectos por los que se ha preguntado (de menos de un punto), por lo que representa de acuerdo en la valoración positiva de la experiencia. Un $50 \%$ o más de los estudiantes han puntuado con un 4 o un 5 todas las características evaluadas. La utilidad de la experiencia en sí misma para el aprendizaje, aspecto que puede ser indicativo globalmente de la satisfacción, tiene una puntuación media de 4,09 puntos.

Los datos cualitativos apuntan en la misma dirección: ante la pregunta de qué mejoras introducirían, un 32\% del alumnado no cambiaría nada de la experiencia. Algunos ejemplos son: "Sinceramente, no sugeriría nada en concreto para mejorar la experiencia. Me ha parecido todo muy correcto", "Continuar como hasta ahora, todo perfecto". El resto de las respuestas sugieren cambios relativos, principalmente, a la longitud del proyecto: aumentar el número de sesiones o que éstas fueran de mayor duración; "Incluiría en la medida de lo posible más sesiones o que dure más el proyecto, ya que fue de gran ayuda y fue increíble participar en ér'. Y algunas sugerencias van en la línea de realizar encuentros presenciales y no solo virtuales entre las tres universidades; "Actividades de participación y convivencia con los alumnos de las otras dos universidades", "Un encuentro presencial final de las tres universidades". Cabe decir que este último aspecto estaba previsto en la planificación inicial, pero fue inviable realizarlo finalmente debido a la situación de pandemia. 
Tal y como se muestra en la figura 5 , los aspectos mejor valorados de la experiencia, considerados de forma individual, son la motivación por seguir aprendiendo después de su participación en la experiencia (Media=4,34), el desarrollo de la creatividad (Media=4,33), la mejora del trabajo colaborativo, de la relación con el profesorado implicado de su universidad, el trabajo conjunto con estudiantes y profesores de otras universidades (Media $=4,30$ ), el progreso en competencias relacionadas con innovación social (crear nuevas ideas, aplicar otras metodologías...) y pensamiento crítico (Media=4,27). Además, en dos de estas dimensiones -la motivación por seguir aprendiendo y la valoración positiva del trabajo interuniversitario- el acuerdo en la evaluación positiva es alto, ya que son las que tienen las desviaciones típicas más bajas $(0,89$ y 0,87 respectivamente).

En estas dimensiones mejor valoradas la puntuación mediana es 5, lo que significa que un 50\% o más de los estudiantes han puntuado con el valor máximo estas características de la experiencia. Están cercanos a los 6 de cada 10 participantes aquellos que están absolutamente satisfechos con las contribuciones de la experiencia al desarrollo de su creatividad (58,5\%), y a su motivación por seguir aprendiendo y la mejora de la relación con los profesores que han participado (55,4\% en ambos aspectos).

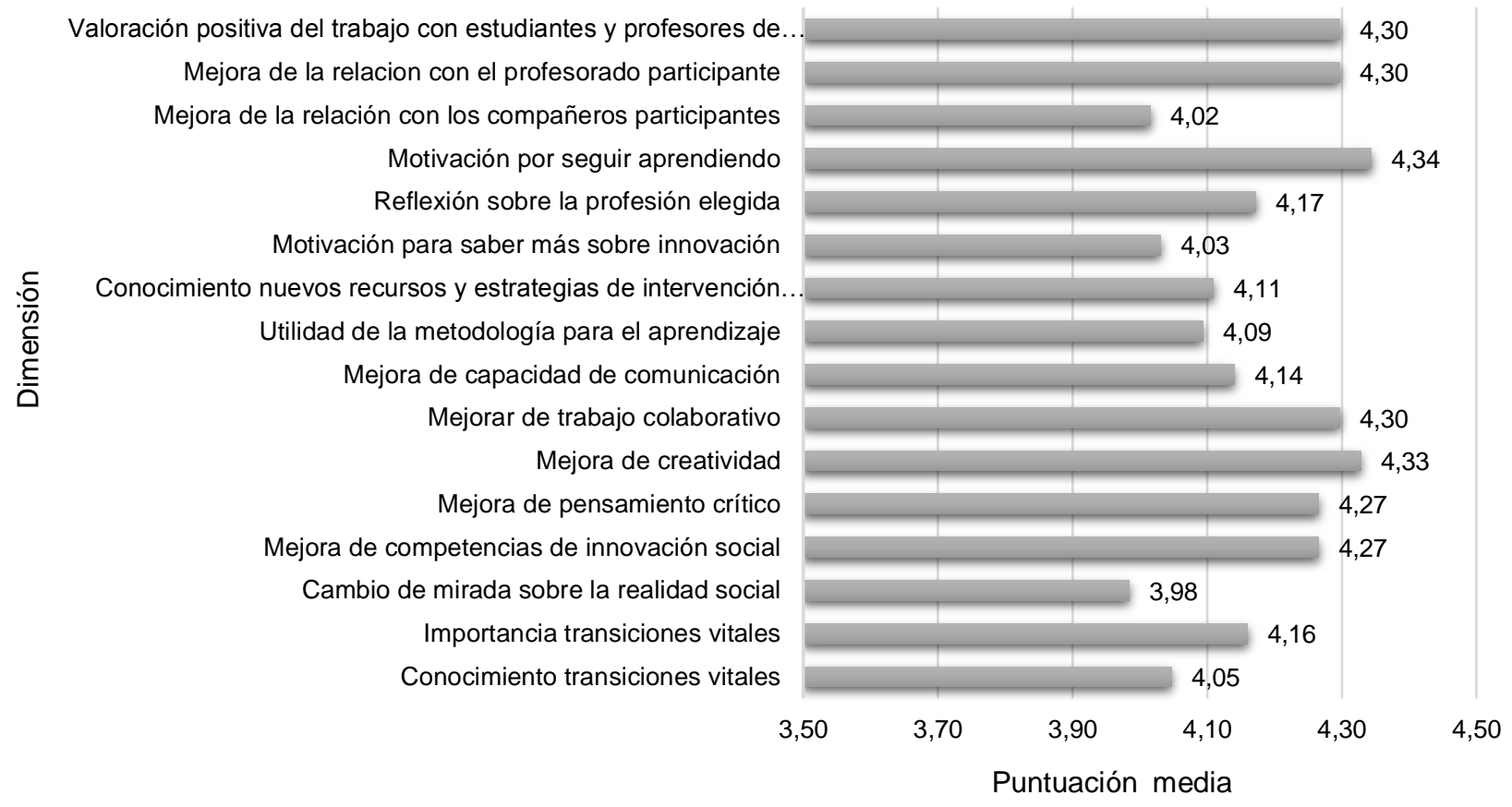

Fig. 5: Puntaciones medias dimensiones evaluadas

Las respuestas de los estudiantes a la pregunta abierta “¿Con qué te quedarías de la experiencia?” refuerzan el análisis cuantitativo expuesto. La motivación al aprendizaje, el desarrollo de la creatividad, el trabajo colaborativo e interuniversitario y el progreso en innovación y pensamiento crítico son también los cuatro aspectos que aparecen con más frecuencia, siendo el desarrollo de la creatividad, mencionado en un $46 \%$ de las respuestas, el más reiterado. A continuación, recogemos algunas frases representativas a modo de ejemplo: (i) "El trabajo en una reflexión más profunda, dinámica y grupal sobre la Educación social y los temas tratados es lo que más destacaría. Mediante la práctica he aprendido mucho más y la motivación ha incrementado a la hora de trabajar"; (ii) "Lo que más me ha gustado de esta experiencia ha sido la metodología utilizada, ya que he descubierto cierta creatividad que pensaba que no tenía". "Me quedo con la forma de tratar de ser creativos a la hora de dar respuestas adaptadas a las nuevas necesidades sociales"; (iii) "De esta experiencia me quedo con todo el trabajo en equipo entre los compañeros, los profesores y el resto de las universidades. Ha sido bonito ver como todos remábamos en la misma dirección y poco a poco se iba dando forma a este proyecto tan especial"; $y$ (iv) "Ha sido una experiencia muy enriquecedora, probablemente me quedo con el avance en mi pensamiento crítico".

Por otro lado, han sido las cuestiones relacionadas con el cambio de mirada sobre la realidad social (Media=3,98), la mejora de la relación con sus compañeros (Media=4,02), y la motivación por saber más sobre innovación (Media=4,03), las que han obtenido valoraciones más bajas. 3 de cada 10 estudiantes puntúan con un $2(7,7 \%)$ o con un $3(23,1 \%)$ el hecho de que la experiencia les haya ayudado a adoptar una nueva perspectiva. La misma distribución existe en relación con que la experiencia haya contribuido a mejorar la relación con sus compañeros, aspecto que el 9,2\% evalúa con un 2 y el $23,1 \%$ con un 3 . El que haya 
incrementado su motivación para saber más sobre innovación es evaluado con un 2 por el 13,9\% de los estudiantes, el grupo más numeroso en esta baja puntuación de todas las características evaluadas. Además, son aspectos que recaban más desacuerdo respecto a su valoración, mostrando una dispersión más alta. La motivación por saber más en innovación tiene la desviación típica más alta (1,01 puntos), seguida de la cuestión de la adopción de una nueva perspectiva sobre la realidad social $(0,97)$.

Teniendo en cuenta la evaluación de la experiencia por áreas (ver Figura 6), los estudiantes tienen una buena autopercepción de logro de competencias de innovación social, y se muestran muy satisfechos con el valor relacional de la iniciativa formativa. El área de innovación agrupa cuatro de los aspectos mejor valorados competencias para la innovación, desarrollo de pensamiento crítico, mejora de la creatividad, y del trabajo colaborativo-, y ha sido el mejor puntuado de los cinco evaluados (Media=4,21). Es ilustrativo que el 92,3\% de los estudiantes también nombran uno o varios de estos cuatro aspectos cuando se les pregunta de forma abierta con qué se quedan de la experiencia. Se confirma que, junto con el área relacional (que aparece en el $32,3 \%$ de las respuestas), es el área de la vivencia educativa la que les ha impactado en mayor medida: "Me quedo con poder haber aprendido nuevas perspectivas de actuación y también el clima formado entre profesores y compañeros". "Me quedo con todas las ideas aportadas sobre innovación y creatividad". "El estilo de trabajo innovador. Creo que muchas veces se deja de lado y es un método de aprendizaje muy útil". "Me encantó todo lo que hicimos en el proyecto, pero sobre todo se agradece salir de la rutina de las clases y hacer proyectos más creativos y que nos abran la mente al futuro".

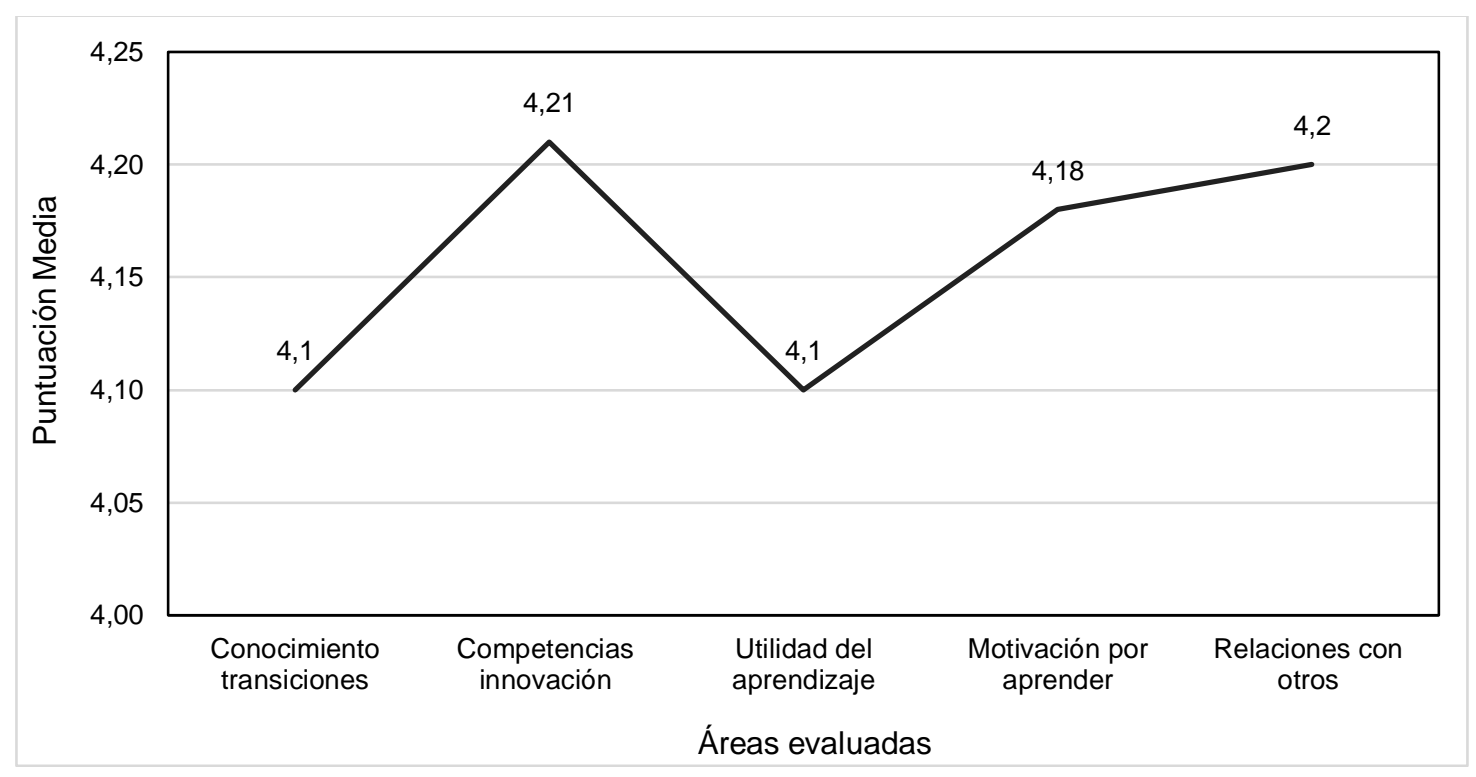

Fig. 6: Puntuaciones medias áreas evaluadas

El área relacional del proyecto recaba también una evaluación muy alta (Media=4,20), además de por la mejora en las habilidades y competencias para el trabajo colaborativo que los estudiantes manifiestan, porque consideran de forma muy positiva la oportunidad que han tenido para relacionarse con el profesorado que los ha acompañado, y el trabajo con estudiantes y profesores de las otras universidades implicadas. Las áreas de la motivación por el aprendizaje y la orientación a la profesión elegida (Media $=4,18$ ), la utilidad de la experiencia para el aprendizaje en general, y el mayor conocimiento que han adquirido sobre las transiciones vitales (Media=4,10 en ambos), han recibido una evaluación más baja, pero en cualquier caso también satisfactoria.

Acabamos este apartado presentando la nube de palabras surgida de la pregunta final del cuestionario: ¿Cuáles son las 3 palabras que asocias a la innovación social?, que puede verse en la figura 7. Como puede apreciarse la palabra más utilizada ha sido creatividad, seguida de aprendizaje, evolución, equipo, motivación, mejora, cambio o progreso entre otras. La elección de la palabra creatividad indica que los participantes han captado con claridad uno de los rasgos esenciales de la innovación social, la capacidad para adoptar nuevas perspectivas y pensar de forma divergente. La aparición de las palabras aprendizaje, evolución, motivación, equipo, hablan de la vivencia positiva de la propia situación de aprendizaje y finalmente las palabras mejora, cambio o progreso, refieren al objetivo final de la innovación social como respuesta, desde el ejercicio profesional, a los problemas sociales. 


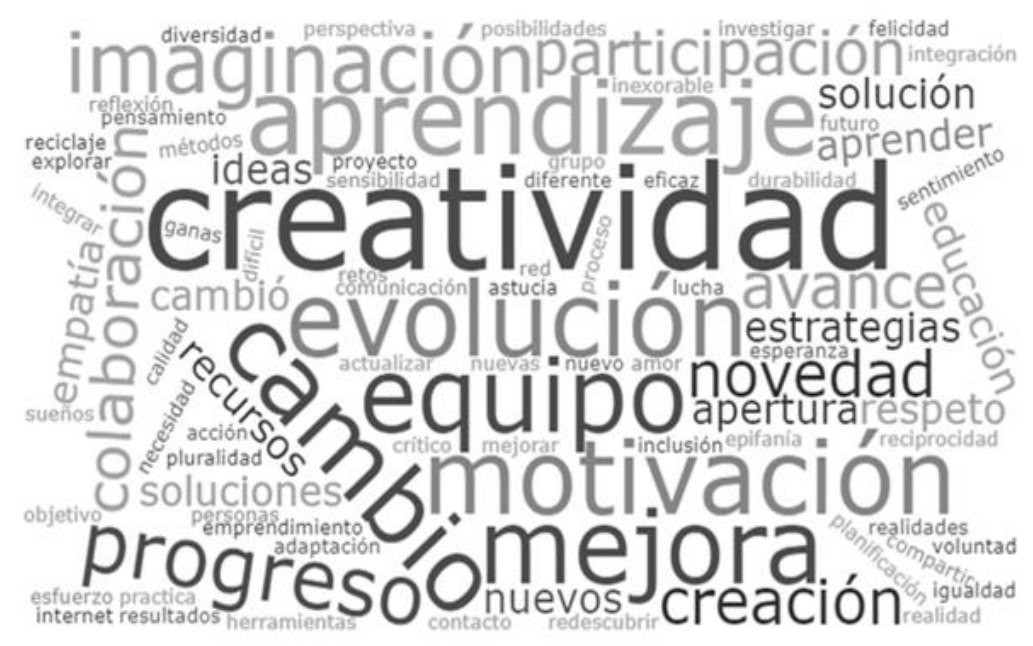

Figura 7: Nube de palabras sobre innovación social

\section{DISCUSIÓN}

Los futuros profesionales de la Educación Social y del Trabajo Social deben incluir la perspectiva de la innovación para afrontar los nuevos retos sociales y modificar las prácticas de intervención con las que abordar las dificultades sociales asociadas a las transiciones vitales. (Howaldt, 2019). En este trabajo se ha presentado una experiencia de aprendizaje de métodos y técnicas para el diseño de propuestas de intervención innovadoras en el acompañamiento a personas que transitan vitalmente por formar o finalizar un proyecto de familia y crianza, empobrecerse, tener o perder un empleo, y vivir procesos de duelo. Constituye una iniciativa universitaria dirigida al desarrollo del pensamiento crítico, la creatividad y el trabajo colaborativo (Alden et al., 2015), orientada a preparar a los futuros profesionales para desarrollar estrategias innovadoras, no de carácter tecnológico, sino en la intervención con los problemas que las personas han de afrontar en su vida cotidiana. Este proyecto formativo ha cumplido satisfactoriamente su objetivo de utilizar técnicas docentes innovadoras para responder creativamente a los entornos de gran complejidad social que caracterizan la sociedad actual. Este es un rasgo esencial de las profesiones consideradas de intervención social (Leal et al., 2016), que han de idear nuevas formas de enfrentar las injusticias sociales y trabajar en pro de la igualdad de derechos y la plena inclusión de toda la ciudadanía. Así se desprende de la valoración positiva que ha recibido por parte del alumnado de Trabajo Social y de Educación Social de las tres universidades participantes, que la han considerado de gran relevancia en su crecimiento profesional.

Para estimular en los estudiantes el desarrollo de propuestas creativas, en esta experiencia interuniversitaria de formación se ha utilizado la técnica de "Design Thinking", que se aleja de las metodologías propias de las clases magistrales, creando espacios de reflexión crítica para, mediante el trabajo en equipo con otros estudiantes y con el profesorado, encontrar alternativas de solución a problemas complejos (Brown, 2008; Melles et al., 2012). El desarrollo de la experiencia es valorado muy positivamente por los estudiantes participantes, destacándose la realización de un trabajo colaborativo horizontal con el profesorado, que ha abierto nuevas oportunidades de relación y de aprendizaje mutuo, a lo que se ha sumado la dimensión interuniversitaria del proyecto.

Por otra parte, las dinámicas de trabajo son percibidas por los estudiantes como valiosas en el desarrollo de la iniciativa y actitud emprendedora, la capacidad de auto-organización para la resolución de problemas, las habilidades de negociación y trabajo en equipo y las competencias comunicativas. Es importante que el alumnado tome un papel activo en los procesos de aprendizaje, motivándole hacia la innovación social (Leal et al., 2019). Esta experiencia ha demostrado que su respuesta es comprometida y positiva cuando se les coloca en el centro del proceso y se les estimula en el desarrollo de nuevas ideas en un clima apropiado de trabajo que impulse su liderazgo. Además, es significativo destacar el impacto que este tipo de aprendizajes innovadores tiene sobre la autopercepción positiva de los estudiantes en su motivación por seguir aprendiendo y creciendo en su elección profesional. Completan así el paradigma pedagógico interactivo y de educación integral, que contempla que el desempeño profesional no se nutre sólo de conocimientos académicos y prácticos, sino que deben acompañarse de una disposición de reflexión, búsqueda y aprendizaje a lo largo de su vida profesional siempre en pro del mayor bien para las personas con las que trabajan.

Es importante resaltar la conexión entre aprendizajes curriculares y la recreación de acciones profesionales reales como elemento crucial de éxito de la experiencia. La lógica formativa del Espacio Europeo de 
Educación Superior centrada en la adquisición de competencias profesionales demanda, por una parte, romper con la parcialización y segmentación de los contenidos en diferentes asignaturas, y por otra, generar conexiones entre el bagaje teórico y la praxis profesional. Por otro lado, este tipo de iniciativas de aprendizaje de innovación social aporta un valor añadido desde la perspectiva del proyecto pedagógico de las tres universidades implicadas. Su modelo se caracteriza por orientar la docencia a la formación de profesionales que sean capaces de cambiar y mejorar el mundo a través de la excelencia en su desempeño, comprometidos con la realidad que les rodea, en especial con aquellas personas más vulnerables, y con una profunda conciencia y reflexividad sobre su acción profesional y su relación con los demás.

La realización de este proyecto abre nuevos retos para dar continuidad a la experiencia en el futuro y que sea replicada por otras Universidades, dada la buena acogida y la evaluación positiva de los estudiantes participantes, a pesar de no haber podido concluirla como estaba previsto. No se debe olvidar que la última etapa del desarrollo de la iniciativa formativa coincidió con el Estado de alarma y el consiguiente confinamiento domiciliario de la población española por la pandemia causada por el COVID-19. Como consecuencia, la docencia universitaria pasó a desarrollarse en un formato online, afectando a la puesta en común prevista en una jornada interuniversitaria, dirigida a compartir las experiencias y conclusiones obtenidas por los diferentes equipos de cada uno de los centros universitarios, dado que el trabajo de los grupos en cada Universidad si pudo llevarse a cabo de forma presencial.

Asimismo, sería interesante incorporar a la experiencia a profesionales en ejercicio que pudieran contrastar y evaluar las propuestas elaboradas por los estudiantes, promoviendo la reflexión sobre su utilidad, viabilidad y posibles mejoras en los procesos y resultados. Esta perspectiva resultaría muy enriquecedora y motivadora por su potencial de conexión entre la esfera académica y la de la práctica profesional. El dialogo con el mundo profesional también representa la vocación de apertura a la sociedad de los procesos de ideación y diseño de prácticas profesionales innovadoras. La valoración positiva de esta experiencia y de las técnicas utilizadas recomienda que este tipo de acciones formativas se incorporen progresivamente en los estudios universitarios de Grado de manera formal, mediante la creación y/o mejora de asignaturas específicas de innovación social, de laboratorios de innovación social o de nuevas metodologías en asignaturas del prácticum. Para dar continuidad a este proyecto, se propone vincularlo de manera formal a algunas asignaturas del currículo en las titulaciones implicadas.

\section{CONCLUSIONES}

Este artículo muestra una experiencia formativa exitosa destinada al estímulo y desarrollo de la creatividad de los futuros educadores y trabajadores sociales en la intervención con personas en circunstancias difíciles asociadas a transiciones vitales como la constitución de una familia, la crianza, el empobrecimiento, los cambios en el empleo, y los procesos de duelo. La recogida de datos sobre el impacto de los elementos claves en la satisfacción de los estudiantes nos ha permitido concluir lo siguiente: 1) la técnica propuesta basada en el "Design Thinking" genera una motivación y percepción muy positiva en los estudiantes sobre nuevas y creativas posibilidades para afrontar retos sociales en entornos complejos; 2) la conexión entre aprendizajes curriculares y la recreación de acciones profesionales reales desde una perspectiva innovadora, se ha erigido como elemento crucial para el éxito de la iniciativa; 3) el protagonismo otorgado al alumnado hace que se implique en el proceso y responda de forma comprometida, desarrollando su iniciativa, capacidad de auto-organización, pensamiento crítico y competencias comunicativas; 4) el trabajo horizontal conjunto profesorado-estudiantes, así como la dimensión interuniversitaria, aporta una riqueza a la experiencia que es percibida y muy valorada por los participantes; 5) se pone de manifiesto que iniciativas formativas que promueven el pensamiento crítico, la ideación creativa, y el trabajo colaborativo ofrecen nuevas perspectivas de formación para futuros profesionales de la acción social, que son percibidas muy positivamente por los propios estudiantes universitarios.

\section{REFERENCIAS}

Alden, B., Armellini, A., y otros tres autores, Social Innovation Education: towards a Framework for Learning Design, https://doi.org/10.1108/HESWBL-04-2015-0026, Higher Education. Skills and Workbased Learning, 5(4), 383-400 (2015)

Alonso, A., y Echevarría, J., ¿Qué es la Innovación Social? El Cambio de Paradigma y su Relación con el Trabajo Social, https://doi.org/10.5209/CUTS.51752, Cuadernos de Trabajo Social, 29(2), 163-171 (2016)

Alonso, L., y Fernández, C., La Innovación Social y el Nuevo Discurso del Management: Limitaciones y Alternativas, https://doi.org/10.3989/arbor.2011.752n6009, ARBOR Ciencia, Pensamiento y Cultura, 187(752), 11331145 (2011)

Bauman, Z., Tiempos Líquidos: Vivir en una Época de Incertidumbre, $2^{\underline{a}}$ ed. Tusquets, México D.F., México (2009)

Bennet, N., y Lemoine, J., What a Difference a Word Makes: Understanding Threats to Performance in a VUCA World, http://dx.doi.org/10.2139/ssrn.2406676, Business Horizons (2014) 
Berzin, S.C., y Catsouphes, M.P., Stimulating Innovation within Social Sector Organizations: The Application of Design Thinking, DOI: 10.4172/2169-026X.1000156, Journal of Entrepreneurship \& Organization Management, 4(3) (2015)

Brown, T., Design Thinking, Harvard Business Review, 86(6), 84-92 (2008)

Edwards-Schachter, M., y Wallace, M. L., 'Shaken, but not Stirred': Sixty Years of Defining Social Innovation, DOI: 10.1016/j.techfore.2017.03.012, Technological Forecasting and Social Change, 119, 64-79 (2017)

Garthwait, C.L., Social Work Education: A Vehicle for Innovative Practice, DOI: 10.7763/IJSSH.2015.V5.559, International Journal of Social Science and Humanity, 5(9), 798-803 (2015)

Hernández-Ascanio, J., Tirado-Valencia, P., y Ariza-Montes, A., El Concepto de Innovación Social: Ámbitos, Definiciones y Alcances Teóricos, CIRIEC-España, Revista de Economía Pública, Social y Cooperativa, ISSN: 02138093, (88), 164-199 (2016)

Holmberg, J., y Robert, K. H., Backcasting: A Framework for Strategic Planning, DOI: 10.1080/13504500009470049, The International Journal of Sustainable Development and World Ecology, 7(4), 291-308 (2000)

Howaldt, J., Rethinking Innovation: Social Innovation as Important Part of a New Innovation Paradigm, en Howaldt, J., Kaletka, C., Schröder, A., y Zirngiebl, M. (eds.) Atlas of Social Innovation. 2nd Volume - A World of new Practices, 1620, Oekom Verlag GmbH, Munic, Alemania, (2019)

Leal, M.T.; Riberas, G., y Rosa, G., Fostering Innovation in Social Work and Social Education Degrees: Multilingual Environment and Tools for Social Change, https://doi.org/10.1186/s41239-016-0031-0, International Journal of Educational Technology in Higher Education, 13, 31 (2016)

Leal, M.T.; López, P., y Navarro-Segura, L., Methodologies to Enhance Innovation Competencies in Social Work Education, https://doi.org/10.1080/02615479.2019.1674801, Social Work Education, 40(3), 367-382 (2019)

Martínez Moreno, R.; Cruz Gallach, H. Blanco, I., y Salazar, Y., La Innovación Social, ¿Prácticas para producir Autonomía, Empoderamiento y Nueva Institucionalidad? https://doi.org/10.3989/ris.2019.77.2.17.022, Revista Internacional de Sociología, 77(2), e126 (2019)

Melles, G., Howard, Z., y Thompson-Whiteside, S., Teaching Design Thinking: Expanding Horizons in Design Education, https://doi.org/10.1016/j.sbspro.2011.12.035, Procedia: Social and Behavioral Sciences, 31, 161-164 (2012).

Millar, C.C.J.M., Groth, O., y Mahon, J.F., Management Innovation in a VUCA World: Challenges and Recommendations, https://doi.org/10.1177/0008125618805111, California Management Review, 61(1), 5-14 (2018)

Mulgan, G., The Process of Social Innovation, https://doi.org/10.1162/itgg.2006.1.2.145, Innovations: Technology, Governance, Globalization, 1(2), 145-162 (2006)

Mulgan, G., Social Innovation: How Societies Find the Power to Change. Policy Press, Bristol, Inglaterra (2019)

Nandan, M., London, M., y Bent-Goodley, T., Social Workers as Social Change Agents: Social Innovation, Social Intrapreneurship, and Social Entrepreneurship, https://doi.org/10.1080/23303131.2014.955236, Human Service Organizations Management, Leadership \& Governance, 39(1), 38-56 (2015)

Nardone, G., Diccionario Internacional de Psicoterapia. Herder, Barcelona, España (2019)

Rosa, G., Navarro-Segura, L., y López, P., El Aprendizaje de las Habilidades Sociales en la Universidad. Análisis de una Experiencia Formativa en los Grados de Educación Social y Trabajo Social, http://dx.doi.org/10.4067/S071850062014000400004, Formación Universitaria, 7(4), 25-38 (2014)

Sathiabalan, M., Saranya, R., Arun Kumar, A., y Amarjeet, S., Volatility, Uncertainty, Complexity and Ambiguity (VUCA) in Context of the COVID-19 Pandemic: Challenges and Way Forward, International Journal of Health Systems and Implementation Research, 4(2), 10-16 (2020)

Sinek, S., Start with why. How Great Leaders Inspire Everyone toTake Action, Penguin books, Londres, Inglaterra (2009)

Soria-Barreto, K., Zuñiga Jara, S., y Ruiz Campo, S., Educación e Intención Emprendedora en estudiantes universitarios: un caso de estudio, http://dx.doi.org/10.4067/S0718-50062016000100004, Formación Universitaria, 9(1), 25-34 (2016)

Staniforth, B., Fouché, C., y O’Brien, M., Still Doing what we Do. Defining Social Work in the $21^{\text {st }}$ Century https://doi.org/10.1177/1468017310386697, Journal of Social Work, 11(2) 191-208 (2011) 